\title{
Unidos por la red, separados por el Estado. Una lectura sobre la inclusión digital en Colombia*
}

\author{
Patricia Martínez Coral ${ }^{* *}$ \\ (patricia.martinez@uexternado.edu.co)
}

Artículo de investigación recibido el 28/11/2014 y aprobado el 19/12/2014

Cómo citar este artículo:

MARTÍNEZ, Coral Patricia (2014). "Unidos por la red, separados por el Estado. Una lectura sobre la inclusión digital en Colombia”. En: Trans-pasando Fronteras, núm. 6, pp. 157-182. Cali, Colombia: Centro de Estudios Interdisciplinarios, Jurídicos, Sociales y Humanistas (CIES), Facultad de Derecho y Ciencias sociales, Universidad Icesi.

\begin{abstract}
Resumen
El presente artículo se propone contribuir al debate teórico sobre la naturaleza institucional de la difusión tecnológica, a la luz de la evidencia encontrada en el desarrollo de las iniciativas de acceso universal a Internet en Colombia, las cuales se enmarcan en la política de telecomunicaciones sociales, vigente desde 1995. Una vez introducidos los fundamentos que orientan la discusión teórica, y su aplicación concreta en el campo de las Tecnologías de la Información y la Comunicación (TIC), se exponen las lecciones más relevantes acerca de los condicionamientos institucionales que se desprenden de la interacción entre Estado y mercado, diseño de intervenciones públicas, y elecciones técnicas,
\end{abstract}

* La ponencia expone algunos de los hallazgos correspondientes a la investigación mediante la cual se participa por el título de Ph.D. en Estudios Políticos.

** Estudiante de Doctorado en Estudios Políticos de la Universidad Externado de Colombia. 
para las oportunidades de acceso a Internet en las zonas rurales del país; en razón de la pertinencia que tiene actualmente la búsqueda de alternativas para viabilizar la vida en el campo, con ocasión de las proyecciones sobre los escenarios de pos-conflicto. Por último se esbozan las conclusiones que permiten desvirtuar algunos paradigmas que revisten las decisiones de acción pública, de las que surge el afianzamiento de la exclusión digital en el país.

\section{Palabras clave:}

Exclusión digital, difusión tecnológica, acceso universal, condicionamiento institucional.

\section{Introducción}

La dimensión política de la tecnología constituye un problema fundamental para las ciencias sociales contemporáneas. A lo largo del proyecto moderno, la disyuntiva entre la neutralidad del cambio tecnológico, postura según la cual las innovaciones técnicas se originan de manera autónoma y sin pretensiones políticas o culturales; y su antítesis política, inspirada en el reconocimiento de los estrechos vínculos existentes entre tecnología y poder, así como en los arreglos institucionales que le dan forma, hizo parte central de las aproximaciones teóricas sobre filosofía de la ciencia. Por su parte, este debate se mantuvo vigente, extendiéndose a diversas disciplinas del conocimiento, durante el siglo XX en razón de las turbulencias socioeconómicas acaecidas con la Revolución Industrial, las dos guerras mundiales, la confrontación ideológica entre el comunismo y el capitalismo, y la amenaza nuclear surgida durante la Guerra Fría. En la actualidad, el desarrollo de las tecnologías de la información y la comunicación (TIC) inclina la balanza en favor de la interpretación crítica del cambio tecnológico.

Tal como argumenta Mumford (2006), la dimensión política de la tecnología se evidenció mucho antes del arribo de la máquina de vapor, ícono de la Era industrial. De acuerdo con el autor, la creación del reloj al interior de los monasterios del imperio romano en el siglo XIII logró introducir un orden ajeno a la naturaleza humana, y la creencia en un mundo independiente y cuantificable; la fuerza creativa de dicho artefacto demostró su capacidad de transformar para siempre el estilo de vida de la sociedad. Su uso generalizado por fuera de los claustros, dotó de regularidad el comportamiento del trabajador y el comerciante, de tal modo que el tiempo se convirtió en espacio cerrado, susceptible de ser dividido, copado, y dilatado mediante el invento de otros instrumentos que proporcionan eficiencia. Una vez perfeccionada la representación del espacio, con el descubrimiento de las leyes de la perspectiva en la pintura y luego en la cartografía, 
durante los siglos XIV y XVII, se inició la exploración del movimiento y se sentaron las bases de las técnicas modernas. En el contexto de la sociedad capitalista, las abstracciones adquirieron gran importancia; el tiempo se convirtió en dinero, el dinero en poder, y las máquinas en un recurso impersonal para el ejercicio del control social.

En complemento de esta descripción, Winner (1980) afirma que la tecnología es, en sí misma, un fenómeno político, debido a las numerosas posibilidades que ofrecen los dispositivos técnicos para ordenar la actividad humana. De forma consciente o inadvertida, las sociedades eligen sistemas tecnológicos que inciden en el desempeño ordinario del trabajo, el consumo, la comunicación y demás procesos que estructuran la toma de decisiones, generando notables diferencias de poder entre los individuos. Desde esta perspectiva, el vector de cambio no reside en la fuerza orientadora de los artefactos, sino en el entorno institucional que hace posible su gestación y desarrollo. Veblen (1914), por ejemplo, observó que el sistema fabril que dejó atrás la organización productiva de los artesanos, fundó un nuevo orden social bajo el amparo de libertades civiles que proclamaron la coincidencia entre el derecho a la ganancia del empresario y la realización de las oportunidades económicas de la comunidad. Sin embargo, estas transformaciones no se habrían logrado, pese a las innovaciones técnicas, de no contar con el acondicionamiento del sistema escolar -que proporcionó los conocimientos indispensables para satisfacer la demanda industrial de mano de obra-y la maduración del derecho natural.

Asimismo ocurre en los procesos de transferencia tecnológica, cuya comprensión parte de diferenciar los productos materiales de la tecnología, de los sistemas de pensamiento que los crea. Esta sugerencia es formulada por Schaniel (1988), con base en su estudio sobre la introducción de objetos de hierro en la cultura Maorí, que permaneció aislada en Nueva Zelanda hasta finales del siglo XVIII, cuando llegaron los primeros visitantes europeos. En la reconstrucción de ese proceso, el autor encontró que el remplazo de los objetos de piedra, hueso y madera se llevó a cabo durante casi un siglo, iniciando por la dotación de armas hasta impactar la agricultura. A lo largo de este recorrido, los nativos también emplearon los artefactos de hierro en usos distintos para los que fueron diseñados originalmente. Esta serie de hallazgos le permitió concluir que la innovación tecnológica genera cambios, pero que éstos están supeditados al contexto cultural e institucional de la sociedad que acoge los productos técnicos; pues la introducción de los mismos es valo- 
rada por el grupo receptor sin que medie contacto alguno con el sistema de creencias en el que surgieron. Lo anterior es consistente con el carácter relativo de las explicaciones sobre el éxito y fracaso de algunas tecnologías, que impide establecer su superioridad o inferioridad intrínseca, en tanto que tal discernimiento obedece a la historia de la adopción y mejoramiento de los dispositivos técnicos (MacKenzie y Wajcman, 1999).

En esta línea, Hughes (1987) define los sistemas tecnológicos como un conjunto compuesto de artefactos físicos; el ambiente natural en el que operan; las organizaciones que los producen, distribuyen, financian, y regulan; así como aquellas que proveen los conocimientos científicos y técnicos requeridos para su diseño e implementación. A su vez, estos sistemas experimentan fases de invención, desarrollo, innovación, transferencia, crecimiento y consolidación, aunque no existe un orden secuencial entre cada una de ellas. Tan pronto como maduran los sistemas, en lugar de adquirir autonomía, alcanzan un estado de inercia producido por la suma de intereses que privilegian su continuidad, teniendo en cuenta el equipo en los que se soportan, las inversiones realizadas, y los hábitos sociales que se desprenden del uso tecnológico. En consecuencia, los sistemas se tornan inflexibles y pueden prolongarse por generaciones hasta la emergencia de una nueva revolución.

El antagonismo latente entre la noción que presenta la tecnología como un factor externo, determinante del orden social, y aquella que la concibe como un producto resultante de las estructuras sociales, aunque puede resultar estéril para la sociología, nutre el análisis político. Harbers (2005) afirma que no es posible establecer una distinción clara entre explanans y explanandum, por lo que ambas categorías deben entenderse como entidades mutuamente constituidas en un mismo, y único, proceso histórico. El dilema que plantea este referente, denominado "co-producción", se traduce en un problema de agencia (capacidad de actuar) que obliga a reflexionar acerca de quiénes, o cuales, son los actores que participan en dicho proceso, y cómo lo hacen. Para Latour (2002), las consideraciones morales que se derivan de este ejercicio no se apoyan en un balance entre fines y medios, puesto que la concepción que atribuye a la tecnología el rol de servir como medio para alcanzar un determinado fin, se desvanece ante la evidencia provista por el uso que los individuos le dan. En otras palabras, el uso de los artefactos técnicos desplaza y modifica la intención que les dio origen y, de manera progresiva, 
los individuos renuevan sus deseos generando un flujo incesante de fines y medios. En este sentido, la cuestión acerca de la naturaleza de la tecnología exige superar su plano instrumental y reconocer que ella, al igual que la moralidad, es una categoría ontológica.

Este llamado representa uno de los principales desafíos para el estudio político de la tecnología, pues su concepción instrumental ha sido preponderante en la edificación teórica, cuyos extremos corresponden a la dominación tecnológica y el empoderamiento. El primer enfoque establece que las necesidades humanas no surgen naturalmente, sino a partir de una construcción cultural, impulsada por mitos, relatos y discursos que transmiten una determinada visión de vida deseable. Así, los diseños que crean, apropian o modifican la producción tecnológica consiguen alterar la distribución de poder, riqueza y/o prestigio en una sociedad (Pfaffenberger, 1992). El segundo postula que la tecnología, antes de actuar como una fuerza de dominación, encauza los procesos a través de los cuales diversos agentes que intervienen en la solución de asuntos colectivos, pueden acceder a los mecanismos de fuerza, coerción, seducción y manipulación, con mejoras de eficiencia, efectividad, y confiabilidad (Brey, 2007). Entre estos polos gravitan las críticas feministas a la jerarquía social impuesta por el diseño tecnológico, con la aspiración de subvertir las relaciones de poder que hacen parte integral del mismo (Wajcman, 2008).

Por su parte, la interpretación acerca del papel que desempeña el Estado en el cambio tecnológico se enmarca en alguna de las vertientes anteriores. Foucault $(1982 ; 1997)$ encuentra que el Estado, a través de sus instituciones, elabora, racionaliza y centraliza verdades, convertidas en conocimiento científico. Este Régime du savoir, que se expresa mediante la violencia ideológica del aparato estatal, legitima las creencias sobre la tecnología y el valor que le es atribuido por la sociedad; con lo cual las estructuras políticas del poder, además de arrogarse la definición del bien común, bajo su lógica totalizante, someten la subjetividad de los individuos dictaminando qué y cómo pensar.

Con ocasión de los programas nucleares desarrollados en Francia durante la Posguerra, Hecht (2001) ilustra cómo fue reconstruida la identidad nacional de dicho país, con importantes repercusiones para su modernización industrial, evidenciando que el poder político de la cultura es inseparable de su esfera material. En esta obra el autor propone dos conceptos para estimar el alcance de la relación entre tecnología y poder: tecno-política, entendida como la práctica de usar o diseñar la tecnología en busca de objetivos 
políticos; y regímenes tecno-políticos, definidos como el ensamblaje institucional que orienta el desarrollo tecnológico y que, en ejercicio de la tecno-política, prescribe una visión específica del orden socio-político.

La apropiación tecnológica realizada por el Estado para organizar el funcionamiento de la administración pública, ha servido como otro núcleo temático en esta corriente de análisis. Al respecto, Ellul (1964) señala que la incorporación de instrumentos técnicos al aparato estatal no solo dio origen al conflicto entre clase política y tecnocracia, despojando a la primera de autoridad y competencias; también transformó la perspectiva política con la que el Estado interviene frente al progreso técnico. Así, mientras el Estado liberal impuso barreras como la circulación de sustancias nocivas para la salud, normas contra la concentración industrial para favorecer la competencia económica, y protección laboral para impedir los abusos generados por las técnicas de eficiencia productiva, con fundamento en valores como la justicia, el Estado contemporáneo se caracteriza por una supresión radical de las consideraciones ideológicas y morales relativas al cambio tecnológico.

El desmantelamiento de los modelos intervencionistas, emprendido en los países industrializados desde los años setenta, ha motivado una profunda renovación de estos enfoques, desvirtuando la capacidad del Estado para controlar los procesos sociales, mediante la canalización de las fuentes de poder político. En este orden de ideas, Beck (1997) identifica que el agotamiento de la modernidad ha propiciado un nuevo escenario, en cual actores externos al sistema político y corporativo protagonizan la toma de decisiones y compiten, desordenadamente, por el poder. Bajo estas circunstancias, el Estado opera como un negociador. En la etapa pos-moderna, agitada por la creciente percepción de riesgos que emergen en remplazo de las instituciones que le dieron forma a la sociedad industrial, las problemáticas relacionadas con tecnología (su diseño, implementación, y efectos) se abren a posibilidades desconocidas. Acogiendo este significado del entorno político vigente, Feenberg (1995) argumenta que la flexibilidad de la arena pública permite confrontar los códigos técnicos -aquellos rasgos que reflejan los valores y creencias hegemónicas que prevalecen en el diseño de la tecnología y que, por el uso cotidiano de la misma, tienden a pasar inadvertidos-; generando con ello una democratización gradual del cambio tecnológico. 
Sin embargo, esta lectura esperanzadora acerca del potencial de la deliberación pública es objetada con base en la noción de epistemología cívica, propuesta por Jasanoff (2007) para explicar que la construcción de la credibilidad en el conocimiento científico no se reduce a las diferencias binarias entre la percepción de expertos y del ciudadano común. Por el contrario, existen diversas formas de conocimiento público, promovidas por la acción de grupos corporativos que confrontan las alternativas formuladas por el Estado, y después de constituir cuerpos de representación cognitiva se convierten en sistemas cerrados que obstruyen la intervención ciudadana. Por ende, el concepto de vida pública solo cobra significado en los procesos de producción y validación del conocimiento tecno-científico, si se lleva a cabo la activación simultánea de las capacidades instrumentales que equilibran los recursos políticos en la sociedad.

\section{Discusiones teóricas en torno a las TIC}

El crecimiento exponencial de las funciones de transmisión, comunicación, procesamiento y almacenamiento de información, promovido por las mejoras en los instrumentos de cómputo -hardware y software-y los avances de las telecomunicaciones, es lo que se conoce como Revolución de las TIC. Sin embargo, desde un punto de vista técnico, la creación de Internet no representa un acontecimiento revolucionario, tal como describe Winston (1998) en su recorrido por el perfeccionamiento de los sistemas eléctricos y electrónicos de la comunicación, desde el origen del telégrafo moderno en la primera mitad del siglo XIX.

Según el autor, el ensayo de las soluciones tecnológicas es antecedido por largas etapas de invención, asociadas con el estado del conocimiento científico, y aun cuando su desempeño resulte exitoso, la fase de difusión permanece sujeta a una extensa serie de condicionamientos institucionales, de los que pueden surgir ajustes y nuevos desarrollos. De esta manera, el diseño de un lenguaje específico para codificar la información complementó los adelantos de la transmisión satelital y la cibernética, alineados con la construcción de redes que han funcionado, a lo largo de la historia, como un patrón en los sistemas de comunicación. Lejos de asimilarse a una invención discreta, Internet surgió de un proyecto de seguridad nacional (Advanced Research Projects Agency Network) fundado en 1957 como respuesta a la incursión soviética en el es- 
pacio. Hacia finales de los años sesenta, la fase de prototipo empezó a consolidarse sin que hubiera, hasta entonces, suficiente número de redes conectadas para impulsar la creación de la red de redes. En 1973 se puso en marcha el correo electrónico internacional; en 1979 se aplicó el primer protocolo de Internet; en 1986 se implantó el sistema de nombres de dominio; y en 1989, con el fin de la Guerra Fría, el proyecto fue clausurado, dando inicio a la etapa de comercialización de Internet, acelerada por la creación de la red informática mundial (World Wide Web).

Ahora bien, el aspecto revolucionario de las nuevas TIC consiste en posibilitar el surgimiento de estructuras alrededor de las cuales se erige un nuevo orden socio-económico, sustentado en el valor de la información y el conocimiento, y la compresión del tiempo y el espacio. Webster (1995) compila tres teorías que coinciden con esta descripción del fenómeno, a saber: (i) La teoría de la Sociedad Pos-industrial (formulada por Bell), que describe cómo el agotamiento del modelo industrial de producción y consumo dio paso a un nuevo sistema ocupacional, liderado por el sector de servicios intensivo en el uso de la información, recurso del que depende un complejo entramado de transacciones económicas. (ii) La teoría de la Escuela Francesa de Regulación que identifica en este periodo de cambio el origen de un nuevo régimen de acumulación capitalista, caracterizado por la creciente integración global de las actividades económicas y financieras que, a través de las TIC, aumentan la productividad y flexibilidad laboral, fortalecen las estrategias de comercialización, y construyen un entorno simbólico que difunde la idea de que el mundo funciona de manera organizada. Y (iii) la teoría de la Sociedad Red (cuyo principal exponente es Castells), en la que se plantea que el desarrollo de redes, por medio de las cuales se reorganizan las actividades socioeconómicas y se construyen identidades colectivas, ha convertido el flujo de información y conocimiento en el factor de mayor valor productivo, generando también nuevas formas descentralizadas de poder.

En oposición a la centralidad de las TIC, prevaleciente en las explicaciones anteriores sobre el cambio social, Stehr (2001) sugiere conceptualizar los procesos socio-culturales que dieron lugar a la creciente demanda de conocimiento. Desde esta perspectiva se reconoce que en las sociedades posindustriales el conocimiento es un fundamento de la autoridad, lo que le convierte en un recurso valioso para librar conflictos políticos. Por ello, el poder direcciona el curso del desarrollo intelectual dejando atrás la orientación de valores para 
promover un conocimiento volcado a la acción; esto permite comprender que en la mayoría de economías desarrolladas, las ciencias naturales y la tecnología superen a las humanidades y las ciencias sociales. Tras la delimitación del nuevo entorno social, se encuentra entonces un proceso gradual de recomposición de las relaciones de poder.

Aunque al comienzo de los años setenta, la percepción sobre la emergencia del nuevo orden socio-económico hacía parte de un amplio consenso intelectual en los países industrializados (Mansell, 2009), el contraste entre las corrientes de la dominación tecnológica y los teóricos del empoderamiento empezaba a abrirse campo en el debate acerca de las implicaciones de aquel contexto de transformación. Touraine (1971) acuñó el término "Sociedad Programada" para advertir que la influencia tecnológica había logrado impulsar la integración de todas las esferas de la vida social, generando nuevas formas de dominación. Así, el estilo de vida de los individuos resulta alineado con el sistema de generación de riqueza, subordinada al consumo y ya no a la producción; los medios de comunicación fortalecen su capacidad de manipular, creando necesidades y actitudes; y el poder político se ejerce introduciendo dependencia respecto de las formas de participación que aletargan la conciencia sobre los conflictos sociales. Este arraigo de la "tecnicidad", en las sociedades posindustriales, es conducido mediante las instituciones educativas, y sustituye los enfoques normativos que tradicionalmente orientaron la conducta social.

Robins y Webster (1988) argumentan que el desarrollo tecnológico producido como alternativa frente a la crisis económica de los años setenta, consiguió flexibilizar el empleo, convirtiendo el tiempo libre en un atractivo comercial. De esta manera se reconfiguraron las microestructuras de la vida cotidiana, y los grandes poderes (corporaciones y agencias gubernamentales) accedieron a instrumentos de control para invadir el cuerpo social mediante la inspección de las preferencias, hábitos, necesidades, transacciones, círculos sociales, y opiniones de los usuarios de las TIC. A partir de entonces, la información y el conocimiento interceptan las relaciones de poder. Por su parte, entre las visiones tempranas de emancipación es preciso destacar la de Toffler (1970), según la cual la transición hacia la sociedad posindustrial corresponde a un periodo de profundización democrática, considerando que las formas políticas en Occidente, a lo largo de la historia, han evolucionado por la demanda de diferenciación social que, a su vez, 
necesita del flujo de una vasta cantidad de información para permitir que individuos y grupos participen en la toma de decisiones y aseguren su respectiva retroalimentación. De este modo, las TIC representan un avanzado mecanismo político, capaz de promover y acelerar dichos procesos.

Las bondades políticas atribuidas a las TIC, en sus orígenes, se sintetizan así: aceleración, diversificación, y reducción de costos de los flujos de información y comunicaciones interactivas, con potencial de incrementar los procesos de participación democrática y mejorar la igualdad de oportunidades socio-económicas; fortalecimiento de la rendición de cuentas y herramientas de consulta y seguimiento a las agencias gubernamentales; promoción de redes cívicas; generación de vínculos sociales, identidades y sentido de pertenencia; facilidades para movilizar y accionar coaliciones políticas; y creación de espacios para la libertad de expresión, en los cuales pierden relevancia las restricciones contra la opinión derivadas del género, raza, y estrato socio-económico (Alberts y Papp, 1997). Todas estas expectativas que llevaron a proclamar a la telefonía móvil e Internet, la informática, los satélites, y demás innovaciones de las telecomunicaciones, como "tecnologías de la libertad", por hacer efectivo un flujo de información sin precedentes, y estimular la interacción humana, quedaron condicionadas a las políticas de regulación e inversión llamadas a garantizar la disponibilidad y acceso, tanto a la infraestructura como a los contenidos digitales (Pool, 1983).

Más de dos décadas han transcurrido desde la irrupción de las TIC, y aún en las discusiones recientes se posa un manto de duda sobre la cristalización de las promesas de cambio social. Ni el análisis estructural (relativo a las relaciones socioeconómicas y fuerzas de poder), ni el epistemológico (interesado en comprender la incidencia de las TIC en las herramientas de pensamiento y/o representación del mundo), propuestos por Garnham (2001) como criterios orientadores para realizar este balance, arrojan evidencia concluyente. Dahlgren (2011) recuerda que el empoderamiento cívico promovido por los medios horizontales de comunicación se ve limitado por las estructuras objetivas que, de modo sistemático, repercuten sobre los recursos culturales que califican la participación.

De otro lado, Dreyfus (2009) ilustra cómo las alternativas que ofrece Internet para mejorar la calidad de vida de sus usuarios, por medio de las innumerables oportunidades que provee la interacción con la realidad virtual, pueden demandar sacrificios 
proporcionales que obligan a reflexionar sobre los efectos inesperados del contacto con la tecnología. A su vez, DiMaggio et al. (2001) examinan una vasta literatura internacional de hallazgos sobre los efectos sociales de Internet, encontrando que no existen diferencias sustanciales en las medidas de participación cívica entre usuarios y no usuarios; así como también hay registros de fuerzas sociales de integración y polarización gestadas en la red. Los autores deducen que el vínculo entre tecnología y sociedad nunca es unidireccional. Por tanto, los cambios que cabe esperar no son más que el resultado de la organización que conjuntamente le dan los actores económicos, sociales y políticos al acceso y uso de Internet.

En parte, la dificultad de acotar las expectativas en torno a la capacidad de Internet para moldear el cambio social se explica por el carácter difuso de su significado. De acuerdo con Agre (1998), además de representar un "meta-medio" de comunicación; un sistema informático (referido a un amplio conjunto de prácticas computacionales); y una fuente simultánea de estándares de políticas y políticas de estandarización, Internet constituye un proceso de descubrimiento social que se adscribe a la adopción tecnológica, de tal suerte que en la medida que se exploran nuevas formas de uso, se profundiza la comprensión de las ideas que motivaron la creación de esta tecnología. Es por ello que el desarrollo y uso de Internet transforman continuamente el discurso social. Estas ideas y discursos tienen, por ende, su propia historicidad, recurren a sus propias metáforas, y se fundamentan en sus propios supuestos.

Sin enmarcarse en alguna concepción específica del potencial transformador de Internet, la problemática del acceso a esta tecnología también ha sido abordada como un imperativo moral. Partiendo de la transversalidad y relevancia del uso de Internet para el desempeño de las actividades cotidianas, Bastian y Haslam (2010) describen el ostracismo cibernético en el entorno de las sociedades interconectadas, como una disrupción del sentido de pertenencia a la comunidad humana. Van Den Hoven y Rooksby (2008) definen la privación del acceso a la infraestructura y servicios de información, como un problema de justicia distributiva, y proponen reconocerlos como un bien primario, a la luz de la teoría Rawlsiana; es decir, un bien universalmente requerido como condición para el bienestar del ser humano. Entre las cuatro categorías de bienes primarios del sistema Rawlsiano (derechos fundamentales y libertades; oportunidades; ingreso y riqueza; 
y bases sociales de la dignidad) los autores ubican la información, entendida como conocimiento y objeto informacional para producir conocimiento, en la primera (libertad), indicando que dicha posición le otorga una prioridad absoluta frente a cualquier otra dimensión sustantiva de la igualdad.

No obstante, la acelerada evolución de Internet dificulta, cada vez más, precisar las dimensiones de la brecha digital. De acuerdo con Van Dijk y Hacker (2003) la brecha digital puede descomponerse en, al menos, cuatro esferas: la barrera motivacional (que consiste en la falta de interés por explorar la tecnología); la falta de acceso material a las conexiones y terminales; la insuficiencia de destrezas digitales; y la ausencia de oportunidades de uso, entendidas como los recursos sociales y cognitivos que hacen operativa la interacción en redes y su aprovechamiento. En el contexto actual, denominado "Internet of Things", en el que un vasto conjunto de redes conformadas por objetos físicos (sensores, vehículos, electrodomésticos, entre otros), estructura procesos de comunicación y genera bases de datos, dando lugar a una intensa fusión entre los flujos de información que circulan por la red y el mundo físico, las manifestaciones del enorme poder de micro-procesamiento e identificación de datos en el estilo de vida de los usuarios de Internet se tornan más diversas y activas. Entretanto, las desigualdades en el acceso y uso de la tecnología se multiplican (Selby, 2012).

Es así como la privación del acceso a la infraestructura de conectividad a Internet, representa la modalidad más aguda de exclusión digital. El reto que comporta para los Estados superar esta dimensión de la brecha, implica la necesidad de conciliar objetivos de política social, instrumentos de regulación, e intervenciones públicas, con dinámicas del mercado y lineamientos impartidos por organismos multilaterales influyentes en la materia. Ante este complejo panorama, las categorías de acceso y servicio universal han adquirido una connotación estratégica en el diseño de las iniciativas de acción pública, de tal forma que la primera ha sido entendida como una intervención transitoria que consiste en habilitar un medio de acceso público al servicio, a una distancia razonable para la población beneficiaria desde su lugar de residencia (y de acuerdo con sus respectivas alternativas de transporte); mientras la segunda corresponde al fin último de la política de inclusión digital, y abarca la disponibilidad del servicio en cada hogar. 
Conforme lo anterior, las iniciativas de acceso universal proporcionan un escenario adecuado para explicar cómo opera el marco institucional en los procesos de difusión tecnológica, y desentrañar los vínculos existentes entre tecnología y poder. No solo porque delimitan las oportunidades de contacto con la tecnología en aquellos segmentos de la sociedad que se enfrentan a la ausencia del mercado, reflejando así el alcance de los criterios distributivos que contempla la acción pública; sino también porque, a lo largo de su ejecución, es posible identificar las fuerzas de impulso y resistencia que afectan el logro de los cometidos de inclusión digital.

\section{Lecciones del Caso Colombiano}

El pasado 27 de agosto la Comisión Primera del Senado llevó a cabo el primer debate al proyecto de Acto Legislativo No 08 de 2014, mediante el cual se propone reformar el Artículo 20 de la Constitución Nacional en los siguientes términos:

\footnotetext{
"Artículo 20. Se garantiza a toda persona la libertad de expresar y difundir su pensamiento y opiniones, la de informar y recibir información veraz e imparcial, disponer de acceso a banda ancha en Internet y fundar medios masivos de comunicación. Estos son libres y tienen responsabilidad social. Se garantiza el derecho a la rectificación en condiciones de equidad. No habrá censura" (Gaceta del Congreso $N^{\circ} 423$ de 2014).
}

Esta iniciativa refleja el replanteamiento tardío de las categorías de acceso y servicio universal, que hasta el momento han sido acogidas como principios orientadores de la intervención pública, a diferencia de otros países en los que éstas han sido incorporadas al sistema normativo en calidad de mandatos y obligaciones. En 2002, a través de una Directiva Común, la Unión Europea reconoció como derecho mínimo de los ciudadanos, al margen de su localización geográfica, contar con la disponibilidad de un servicio público de Internet a un precio asequible. En 2008 Suiza introdujo la conectividad a Internet en el alcance básico del servicio universal, implementando un plan coordinado con carriers, compañías de electricidad, y operadores de cable, para desarrollar redes FTTH (Fiber To The Home); la conexión inicial se fijó en velocidades de subida y bajada de 600/100 Kbps, respectivamente. En Estados Unidos, el Plan Nacional de Banda Ancha, aprobado 
por la Comisión Federal de Comunicaciones en 2010, incluyó la meta de completar la disponibilidad del servicio para toda la población en 2020, iniciando con una velocidad de bajada de 4 Mbps y una velocidad de subida de 1 Mbps; y en 2010 Finlandia consagró el acceso a Internet como un derecho fundamental del ciudadano, indicando que la conexión a cada hogar iniciaría con 1 Mbps, hasta lograr 100 Mbps en 2015 (García, 2012).

$\mathrm{Al}$ margen de la suerte que pueda correr esta propuesta legislativa, durante su tránsito por el Congreso, y en virtud del interés que despierta en la opinión pública la coyuntura de las negociaciones de paz para idear escenarios sociales de pos-conflicto, resulta pertinente indagar acerca de los principales retos de intervención pública que plantea la problemática de inequidad en el acceso a Internet, con particular atención sobre las condiciones que enfrentan las comunidades rurales del país; considerando que (i) respecto de los países industrializados, el atraso de la inclusión digital en Colombia es ostensible; y (ii) la brecha entre las condiciones de acceso a Internet entre la población urbana y rural, en Colombia, es muy amplia.

En la medición del índice de Desarrollo de las TIC -compuesto por indicadores de acceso (suscripciones de telefonía fija, móvil, ancho de banda, y penetración de computadores por hogar); uso (suscriptores y usuarios de banda ancha fija y móvil); y capacidades (tasas de alfabetización digital, y nivel de matrícula en educación secundaria y terciaria)-, el país se encuentra 0.15 puntos por debajo de la media mundial (4.35 en una escala de 0 a 1), situándose en posiciones inferiores a las de Panamá, Uruguay, Chile, Argentina, Costa Rica, y Brasil. En términos de calidad, estimada a partir de las velocidades de conexión, mientras en los países industrializados más del 60\% de las suscripciones a Internet banda ancha son de alta velocidad (mínimo $10 \mathrm{Mbit} / \mathrm{s}$ ), dicho porcentaje no supera $10 \%$ en Colombia (ITU, 2013). A su vez, la asequibilidad es un factor diferencial relevante. Según Galperin (2013), el precio de una conexión de al menos 2,5 Mbps cuesta casi el doble en Colombia que en los países de la Organización para la Cooperación y el Desarrollo Económico -OECD- (\$50 Vs. 27,2 USD PPP).

En relación con las diferencias de acceso a Internet entre población urbana y rural, la última encuesta sectorial del DANE, correspondiente a los indicadores básicos de tenencia y uso de las TIC, señala que mientras 50,6\% de los hogares en 2013 poseía computador de escritorio, portátil o tableta en las cabeceras municipales, este registro 
ascendía a tan solo $11,7 \%$ en las zonas rurales. De igual modo, la cantidad de hogares urbanos con conexión a Internet en cabeceras era de $43.6 \%$, y en zonas rurales de $6.8 \%$ (DANE, 2014). Estas cifras, si bien desconocen las dimensiones de uso que convergen en el alcance multidimensional de la brecha digital, tales como las habilidades y conocimientos para el manejo de hardware y software, dominio de lenguas extranjeras predominantes en los contenidos virtuales, y demás destrezas necesarias para aprovechar los recursos de la red, ilustran la severidad de la exclusión social que enfrentan a diario las comunidades rurales del país.

Asimismo, en razón de las limitaciones de oferta y demanda que obstaculizan la comercialización del servicio de conectividad a Internet en las zonas rurales, la búsqueda de lineamientos de acción pública debe enmarcarse en las lecciones aprendidas a lo largo de la ejecución de los proyectos de acceso universal, derivados de la política de telecomunicaciones sociales, creada en 1995 por los cambios de liberalización sectorial; y en virtud de la cual el servicio de telefonía pública en zonas rurales pasó de un esquema de prestación pública directa (a cargo de la Empresa Nacional de Telecomunicaciones TELECOM) a uno de intermediación, dirigido por el Ministerio de Comunicaciones (actual Ministerio TIC), con la expectativa de inducir la oferta del mercado.

Lo anterior teniendo en cuenta que al cierre de 2013, solo 14\% de los Centros Poblados del país disponía de una solución de acceso comunitario a Internet (DANE, 2013), cuya distribución no responde a criterios de equidad. Tomando como referencia la propuesta formulada en el Informe Nacional de Desarrollo Humano (PNUD, 2011) para interpretar el grado de ruralidad de los municipios colombianos, con base en la densidad demográfica y la distancia en línea recta promedio desde un municipio hasta la ciudad grande más cercana (con rangos de población mayor a 1 millón de habitantes; entre 400.000 y 1 millón; 200.000 y 400.000; y 100.000 y 200.000), 77\% de los centros de acceso comunitario a Internet se encontraban localizados en municipios de ruralidad media, $18 \%$ en municipios de ruralidad baja, y solo $5 \%$ en municipios de ruralidad alta. 


\section{Gráfica 1.}

Distribución geográfica de los puntos de acceso comunitario a Internet (2013)

\section{Grado de Ruralidad}
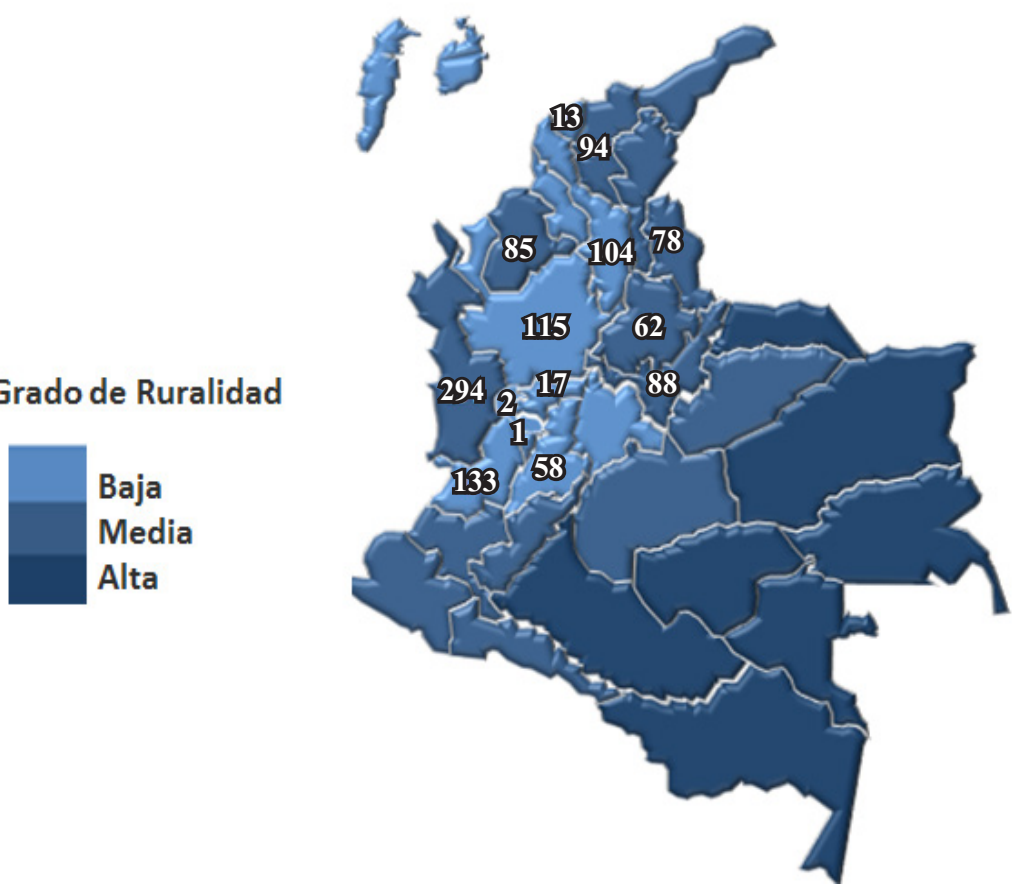

Fuente: Elaboración propia con base archivos de Fondo TIC y el índice de ruralidad del INDH 2011

\section{Lección No 1. La fe en el mercado, más allá de la racionalidad económica, conduce a intervenciones públicas ineficaces e insostenibles}

Como resultado del proceso de liberalización del sector de las telecomunicaciones, emprendido a comienzos de los años noventa, y reforzado mediante la promulgación de la Ley 142 de 1994, "Por la cual se establece el régimen de los servicios públicos domiciliarios y se dictan otras disposiciones", y la ratificación del Acuerdo General de Servicios contenido en la adhesión de Colombia a la Organización Mundial del Comercio (Ley 170 de 1994), la prestación de servicios de telefonía en zonas rurales, que hasta entonces había sido provista por la Empresa Nacional de Telecomunicaciones (TELE$\mathrm{COM}$ ), empezó a regirse por los principios de competencia bajo la coordinación del Fondo de Comunicaciones (unidad administrativa especial adscrita al Ministerio de Comuni- 
caciones). En 1999, a través del Documento CONPES No 3032, fue creado el Programa COMPARTEL para formular y contratar iniciativas de acceso universal, financiadas por el Fondo con cargo a las contraprestaciones que pagan los operadores del sector por concepto de licencias, permisos, concesiones, y multas, entre las cuales se incluyeron las soluciones de acceso comunitario a Internet.

Una década más tarde, con la promulgación de la Ley 1341 de 2009, "Por la cual se definen principios y conceptos sobre la sociedad de la información y la organización de las Tecnologías de la Información y las Comunicaciones-TIC-, se crea la Agencia Nacional de Espectro y se dictan otras disposiciones", este diseño institucional fue confirmado, así como la preponderancia del acceso universal en la delimitación del alcance del Fondo de Comunicaciones (en adelante Fondo TIC):

“(Art. 34) El objeto del Fondo es financiar los planes, programas y proyectos para facilitar prioritariamente el acceso universal, y del servicio universal cuando haya lugar a ello, de todos los habitantes del territorio nacional a las Tecnologías de la Información y las Comunicaciones, así como apoyar las actividades del Ministerio y la Agencia Nacional Espectro, y el mejoramiento de su capacidad administrativa, técnica y operativa para el cumplimiento de sus funciones" (Diario Oficial No. 47426 de julio 30 de 2009).

Ahora bien, las directrices establecidas en el Documento CONPES No 3032 de 1999 señalan que las intervenciones del Programa COMPARTEL estarían orientadas hacia la promoción de la competencia en el sector de las telecomunicaciones, y la vinculación de actores privados para la extender la oferta del mercado, de tal forma que el modelo de inversión a desarrollar consistiría en dejar en manos de los operadores contratados mediante procesos de selección objetiva, la propiedad de la infraestructura instalada para garantizar con ello una rentabilidad suficiente en relación con los costos del montaje, operación y mantenimiento de los puntos de acceso comunitario. Asimismo se acogió la necesidad de promover el uso de tecnologías que permitieran incrementar la eficiencia técnica a costos razonables.

En consonancia con estos lineamientos, los proyectos de acceso universal han sido ejecutados a través de un modelo atípico de inversión pública, denominado contrato de aporte, en el que un tercero se obliga a desplegar, operar, y mantener la infraestructura 
conservando para sí la propiedad de la misma, con el fin de explotarla comercialmente; y los recursos de fomento provistos por el Estado, a través de una asignación modal, son administrados por un encargo fiduciario. De otro lado, las características geográficas, la deficiencia de infraestructura complementaria (energía eléctrica y vías terrestres), los riesgos en materia de seguridad y la baja densidad de población en las zonas rurales, han sesgado el diseño técnico de estos proyectos hacia el uso de soluciones satelitales.

En consecuencia, al término de cada proyecto no ha sido posible lograr el cometido de expandir la oferta del mercado, en tanto que la capacidad de las soluciones satelitales no es escalable y las suscripciones al servicio de Internet, por medio de esta tecnología, son más costosas. Este esquema también conlleva un alto riesgo para la disponibilidad del servicio, debido a la elevada concentración del mercado de proveedores de capacidad satelital, con quienes negocian los intermediarios que prestan el servicio en los proyectos de acceso comunitario. De acuerdo con la Agencia Nacional del Espectro (2013), dicho segmento está constituido por cinco proveedores (67,7\% por Intelsat; $15 \%$ por Telesat; $13,8 \%$ por Satmex; $2,6 \%$ por SES; y $0,9 \%$ por Hispasat), y los programas sociales (aquellos financiados por el Fondo TIC, el Ministerio de Educación Nacional, entre otras instituciones públicas) representan el 29\% de la demanda de capacidad, después de las troncales de tráfico (46\%); seguidas por empresas $(22,6 \%)$, servicios de televisión $(1,3 \%)$, y el sector defensa $(1,1 \%)$.

\section{Lección No 2. El sesgo tecnológico en las intervenciones públicas actúa como fac- tor de la exclusión digital}

A lo largo de la implementación de los proyectos de acceso universal, desde el origen del Programa COMPARTEL hasta el cierre de 2013, se observa que la cobertura rural, tomando como referente el promedio de centros poblados registrados por el DANE en los últimos 6 años (8.077), no ha superado 14\%. Por su parte, las velocidades de descarga, contratadas mediante tecnología satelital, iniciaron en 4 kbps en 2003, aumentaron a 64 kbps en 2006; 128 kpbs en 2008; 256 kbps en 2010; hasta alcanzar 512 kbps desde 2011. Por tanto, el uso de Internet en los centros de acceso comunitario ha estado restringido al envío de correos electrónicos, llamadas telefónicas, navegación simplificada, y descarga de archivos de bajo contenido. Las aplicaciones interactivas, tales como juegos y confe- 
rencias en línea, y la reproducción y/o descarga de videos de alta definición, desbordan la capacidad disponible.

La regulación vigente (Resolución No. 3067 de 2011 de la Comisión de Regulación de Comunicaciones) excluye la definición de banda ancha (capacidad suficiente para permitir, de manera simultánea, la provisión de voz, datos y video, con unas velocidades efectivas de acceso mínimas de 1024 Kbps Downstream y 512 Kbps Upstream, y 1024 $\mathrm{Kbps} / 256 \mathrm{Kbps}$ en el caso de proveedores satelitales) de los contratos suscritos en desarrollo de la política de telecomunicaciones sociales, indicando que para ellos se mantiene como definición regulatoria de banda ancha una relación de 512 Kbps Downstream, y $128 \mathrm{Kbps}$ Upstream para las conexiones satelitales (CRC, 2011).

De otro lado, con base en la relación entre las tarifas al público definidas en cada uno de los contratos celebrados en el marco de los proyectos de acceso universal, y la capacidad de pago de los habitantes rurales (asumiendo que la totalidad del ingreso disponible para servicios de comunicaciones se destinan al uso de Internet), es posible concluir que el número máximo de horas durante el mes para acceder al servicio en los centros comunitarios, ha pasado de 3,1 horas en sus inicios, a 10,7 horas en 2013. En síntesis, la cobertura, calidad, y asequibilidad de los servicios provistos en zonas rurales, han dificultado el desarrollo sostenido de procesos de adopción tecnológica, prolongando las condiciones de exclusión digital en el campo colombiano.

\section{Lección No 3. La efectividad de las intervenciones públicas exige criterios de equidad}

Los primeros proyectos de acceso comunitario a Internet, formulados por el Programa COMPARTEL, estuvieron dirigidos a las cabeceras municipales con población superior a 8.000 habitantes. Una vez se incursionó en zonas rurales, los criterios de selección de las comunidades a beneficiar quedaron expuestos al balance técnico y económico de los proponentes que concurren en los procesos licitatorios del Fondo TIC, sin que primen elementos diferenciadores de la ruralidad, a partir de los cuales se ponderen las necesidades por satisfacer. Esta situación se agudiza aún más por la precariedad de información oficial acerca de las condiciones de vida, rasgos demográficos, disponibilidad de infraestructura y demás factores indispensables para un diseño eficaz de intervención pública. 


\section{Lección No 4: Los resultados de las intervenciones públicas no pueden comprenderse únicamente a partir del contraste entre sus objetivos e instrumentos. Es indispensable incluir en su valoración los rasgos del comportamiento institucional.} La participación del Programa COMPARTEL en los recursos de inversión del Fondo TIC, en promedio, ha sido de $27 \%$. Además de competir por dichos recursos con otras iniciativas del Ministerio, y pese a la prioridad del acceso universal con que fue concebido el Programa, la distribución del gasto ha obedecido a múltiples y cambiantes criterios, de acuerdo con las metas del gobierno de turno. Así, por ejemplo, además de la instalación y operación de puntos de acceso comunitario a servicios de telefonía e Internet, en zonas urbanas y rurales, COMPARTEL ha desarrollado proyectos de conectividad a instituciones públicas en beneficio de bibliotecas, escuelas, alcaldías, juzgados, y guarniciones militares; reposición de redes de telefonía; masificación de accesos a usuario final; y despliegue de infraestructura de transporte. Por consiguiente, la variedad de proyectos, al interior del Programa y del Fondo TIC, representa un elevado costo de oportunidad para la población rural.

\section{Reflexiones finales}

Los hallazgos encontrados a lo largo del repaso por la implementación de la política de telecomunicaciones sociales, permiten comprobar que el modelo de intervención pública adoptado por el Fondo TIC, no ha logrado estimular la oferta comercial de servicios en zonas rurales, desvirtuando los supuestos que fundamentaron la adecuación de los objetivos de acceso universal al régimen de libre competencia; el sesgo tecnológico ha afianzado la problemática de exclusión digital; y la interferencia política en la distribución del gasto público ha obrado en perjuicio de la adopción tecnológica de la población rural.

Lo anterior significa que, en aras de la equidad social, es necesario reconocer las falacias de los paradigmas que inciden en el curso de la acción estatal o, en su defecto, en la estructuración del discurso oficial, a saber: (i) las fallas de mercado no se corrigen sin que la intervención pública sea mediada por una respuesta asertiva de los privados que capturan incentivos. Un modelo de intervención directa, dirigido a sustituir el mercado en un entorno en el que éste no tiene posibilidades de emerger, no solo ofrece mayor eficiencia para la inversión pública, sino mejores resultados en el logro de sus objetivos 
sociales. (ii) Por su parte, la evidencia observada a lo largo de la ejecución de los proyectos de acceso público a Internet, demuestra que la tecnología no es neutral en sus efectos sociales y políticos. En realidad, las decisiones que se amparan en considerandos técnicos entrañan profundas y complejas dinámicas de poder. (iii) Por último, se concluye que los procesos de difusión tecnológica no emergen espontáneamente; son el resultado del arreglo institucional que los rodea.

Los efectos esperados de la aceleración y reducción de costos de los flujos de información y conocimiento, sin duda podrían contribuir a la viabilidad y sostenimiento de un escenario real de pos-conflicto. Especialmente porque las alternativas de progreso socio-económico que en el pasado ofreció la migración del campo a la ciudad, se han reducido en la medida que las estructuras industriales ya no cuentan con capacidad para absorber mano de obra no calificada, tras haber sido desplazadas por sectores intensivos en el uso y procesamiento de información; lo que ha convertido a la inclusión digital en un requerimiento básico, tanto para la viabilidad del campo, como para la continuidad de los procesos de urbanización. Sin embargo, cristalizar las expectativas de empoderamiento, progreso, y libertad, asociadas con el acceso y uso de Internet en las zonas rurales, demanda transformaciones sustanciales que empiezan por reconstruir las bases de la justicia y la equidad.

\section{Bibliografía}

ALBERTS, D. y Papp, D. (1997). Information Age: An Anthology on Its Impact and Consequences. Washington: Ccrp Publication Series.

AGENCIA NACIONAL DEL ESPECTRO (2013). Perspectivas del mercado satelital colombiano. Bogotá: ANE (Subdirección de Gestión y Planeación Técnica del Espectro).

AGRE, P. (1998). "The Internet and public discourse". First Monday, 3 (3-2). 1-7.

ASAMBLEA GENERAL DE LAS NACIONES UNIDAS - ONU. Resolución A / $H R C / 20 / L .13$. Disponible en: http://www.un.org/es/ga/documents/index.shtml

BARÓN, L. F. y Gómez, R. (2014). Más que teclas y pantallas. Acceso público a TIC en Colombia. Cali: Universidad Icesi. 
BASTIAN, B. y Haslam, N. (2010). "Excluded from humanity: The dehumanizing effects of social ostracism”. Journal of Experimental Social Psychology, 46: 107-113.

BECK, U. (1997). "La reinvención de la política: hacia una teoría de la modernización reflexiva". En: Beck, U.; Giddens, A. y Lash, S. (eds.) Modernización Reflexiva, Política, Tradición, y Estética en el Orden Social Moderno. Madrid: Alianza Editorial. Pp. 13-74.

BREY, P. (2007). "The Technological Construction of Social Power". Social Epistemology, 22(1): 71-95.

COMISIÓN DE REGULACIÓN DE COMUNICACIONES (2011). Resolución No. 3067, "Por la cual se definen los indicadores de calidad para los servicios de telecomunicaciones y se dictan otras disposiciones”. Bogotá, CRT.

DAHLGREN, P. (2011). Reinventing Participation: Civic Agency and the Web Environment. At the "Italien Association of Sociology conference on Communication and Civic Engagement”, Sapienza University, Rome, 23-24 Sept. 2011.

DEPARTAMENTO ADMINISTRATIVO NACIONAL DE ESTADÍSTICA (2013), Codificación de la División Político-administrativa de Colombia-Divipola- 2013, Bogotá, DANE.

DEPARTAMENTO ADMINISTRATIVO NACIONAL DE ESTADÍSTICA (2014). Indicadores Básicos de Tenencia y Uso de Tecnologías de la Información y Comunicación en Hogares y Personas de 5 y más años de edad 2013. Bogotá: DANE. Disponible en: https://www.dane.gov.co/files/investigaciones/boletines/tic/bol_tic_2013.pdf

DEPARTAMENTO NACIONAL DE PLANEACIÓN (1999). Documento CONPES No 3032, “Programa Compartel de Telefonía Social 1999-2000”. Bogotá: DNP.

DIMAGGIO, P., Hargittai, E., Neuman, R., y Robinson, J. (2001). Social Implications of the Internet. Annual Review of Sociology, 27: 307-336.

DREYFUS, H. (2009). On the Internet. New York: Routledge. 2nd Edition.

ELLUL, J. (1964). The Technological Society. New York: Random House. 
FEENBERG, A. (1995). Alternative Modernity: The Technical Turn in Philosophy and Social Theory. Oakland: University of California Press, 1995.

FOUCAULT, M. (1982). “The Subject and Power”. Inquiry, 8 (4): 777-795

FOUCAULT, M. (1997). Ethics, subjectivity and truth. New York: The New Press.

GACETA DEL CONGRESO N 423 de 2014, Bogotá.

GALPERIN, H. (2013). "Los precios de la conectividad en América Latina y el Caribe. Reporte 2013". Documento de Trabajo No 15, Centro de Tecnología y Sociedad, Buenos Aires.

GARNHAM, N. (2001). The Information Society: Myth or Reality?. At The "Bugs, Globalism and Pluralism" Conference, Montreal September 19-22, 2001.

GÓMEZ, R. y BARÓN, L. F. (2010). “Acceso público a Internet y cambio social: la experiencia en El Carmen de Bolívar, entre el silenciamiento y la esperanza". En: Revista CS, núm 6, pp. 221-253. Cali: Universidad Icesi

HARBERS, H. (2005). Co-Production,Agency,and Normativity. In: Inside the Politics of Technology. In Harbers, H. (Ed.) Agency and Normativity in the Co-Production of Technology and Society. Amsterdam: Amsterdam University Press. Pp. 9-25.

HECHT, G. (2001). Technology politics and national identity in France In Allen, M. y Hecht, G. (Ed.) Technologies of Power: Essays in Honor of Thomas Parke Hughes and Agatha Chipley Hughes. Cambridge: MIT Press. Pp. 253-293.

HUGHES, T. (1987). The Evolution of Large Technological Systems In: BiJker, W., Hughes, T. y Pinch, T. (Ed.) The Social Construction of Technological Systems. New Directions in the Sociology and History of Technology. Cambridge: M.I.T. Press. Pp. 51-82.

INTER-AMERICAN COMMISSION ON HUMAN RIGHTS (2000). Declaration of Principles on Freedom of Expression. Washington, available: http://goo.gl/9d60hO

INTERNATIONAL TELECOMMUNICATION UNION. (2005). WSIS Golden Book. Geneva: ITU. 
INTERNATIONAL TELECOMMUNICATION UNION. (2013). Medición de la sociedad de la información 2013. Disponible en: :http://www.itu.int/en/ITU-D/Statistics/Documents/publications/mis2013/MIS2013-exec-sum_S.pdf

JASANOFF, S. (2007). Designs on Nature: Science and Democracy in Europe and the United States. New Jersey: Princeton University Press.

LATOUR, B. (2002). Morality and Technology: The End of the Means. Theory, Culture y Society, 19(5/6): 247-260.

Ley No 142 de 1994, "Por la cual se establece el régimen de los servicios públicos domiciliarios y se dictan otras disposiciones”, Bogotá, Diario Oficial 41.433 del 11 de julio de 1994.

Ley No 170 de 1994, "Por medio de la cual se aprueba el Acuerdo por el que se establece la Organización Mundial de Comercio (OMC) suscrito en Marrakech (Marruecos) el 15 de abril de 1994, sus acuerdos multilaterales anexos y el Acuerdo Plurilateral anexo sobre la Carne de Bovino”, Bogotá, Diario Oficial No. 41.637 de 16 de diciembre de 1994.

Ley 1341 de 2009, "Por la cual se definen principios y conceptos sobre la sociedad de la información y la organización de las Tecnologías de la Información y las Comunicaciones-TIC-, se crea la Agencia Nacional de Espectro y se dictan otras disposiciones", Bogotá, Diario Oficial No. 47426 de julio 30 de 2009.

MACKENZIE, D. y Wajcman, J. Eds. (1999) The social shaping of technology. Buckingham: Open University Press. 2nd ed.

MANSELL, R. Ed. (2009). The information society. Critical concepts in sociology. London: Routledge.

MUMFORD, L. (2006). Técnica y civilización. Madrid: Alianza Editorial.

PFAFFENBERGER, B. (1992). "Social anthropology of technology". Annual Review of Antropology, 21: 491-516.

POOL, I. (1983). Technologies of Freedom. Cambridge: Harvard University Press.

PROGRAMA DE LAS NACIONES UNIDAS PARA EL DESARROLLO (2011), Infor- 
me Nacional de Desarrollo Humano 2011, Colombia rural. Razones para la esperanza. Bogotá, PNUD.

ROBINS, K. y Webster, F. (1988). “Cybernetic Capitalism: Information, Technology, Everyday Life". In: The Political Economy of Information, Mosko, V. y Wasko, J. (Eds). Madison: The University of Wisconsin Press. Pp. 45-75.

SCHANIEL, W. (1988). "New Technology and Culture Change in Traditional Societies". Journal of Economic Issues, XXII (2): 493-498.

SELBY, J. (2012). “Anyone's Game: Economic and Policy Implications of the Internet of Things as a Market for Services”. Communications and Strategies 87 (3 Q): 21-40.

STEHR, N. (2001). The Fragility of Modern Societies: Knowledge and Risk in the Information Age. Thousand Oaks: SAGE Publications Ltd.

TOFFLER, A. (1970). Future Shock. New York: Random House.

TOURAINE, A. (1971). The Post-industrial society, Tomorrow's Social History: Classes, Conflicts and Culture in the Programmed Society. New York: Random House.

VAN DEN HOVEN, J. y Rooksby, E. (2008). "Distributive Justice and the Value of Information: A (Broadly) Rawlsian Approach". In: Van Den Hoven, J. y WECKERT, J. (Eds.) Information Technology and Moral Philosophy. New York: Cambridge University Press. Pp. 367-396.

VAN DIJK, J. y Hacker, K. (2003). “The Digital Divide as a Complex and Dynamic Phenomenon". The Information Society, 19: 315-326.

VEBLEN, T. (1914). The instinct of workmanship and the state of the industrial arts. New York: The Macmillan Company.

WAJCMAN, J. (2008). "The Gender politics of technology". In: Goodin, R. y Ti1ly, C. (Ed.)The Oxford handbook of contextual political analysis. New York: Oxford University Press. Pp. 707-721.

WEBSTER, F. (1995). Theories of the Information Society. New York: Routledge. 
WINNER, L. (1980). “Do Artifacts Have Politics?”. Daedalus, 109 (1): 121-136.

WINSTON, B. (1998). Media, Technology and Society: A history from the telegraph to the Internet. New York: Routledge. 